\title{
Frozen section is superior to imprint cytology for the intra-operative assessment of sentinel lymph node metastasis in Stage I Breast cancer patients
}

\author{
Miki Mori ${ }^{1}$, Keiichiro Tada*1, Motoko Ikenaga ${ }^{2}$, Yumi Miyagi ${ }^{1}$, \\ Seiichiro Nishimura ${ }^{1}$, Kaoru Takahashi ${ }^{1}$, Masujiro Makita ${ }^{1}$, Takuji Iwase ${ }^{1}$, \\ Fujio Kasumi ${ }^{1}$ and Mituru Koizumi ${ }^{3}$
}

\author{
Address: ${ }^{1}$ Department of Breast Surgery, Cancer Institute Hospital, Tokyo, Japan, ${ }^{2}$ Department of Cytology, Cancer Institute Hospital, Tokyo, Japan \\ and ${ }^{3}$ Department of Radiology, Cancer Institute Hospital, Tokyo, Japan \\ Email: Miki Mori - miki.mori@jfcr.or.jp; Keiichiro Tada* - ktada@jfcr.or.jp; Motoko Ikenaga - motoko.ikenaga@jfcr.or.jp; \\ Yumi Miyagi - yumi.miyagi@jfcr.or.jp; Seiichiro Nishimura - snishimura@jfcr.or.jp; Kaoru Takahashi - kaoru.takahashi@jfcr.or.jp; \\ Masujiro Makita - mmakita@jfcr.or.jp; Takuji Iwase - takuji.iwase@jfcr.or.jp; Fujio Kasumi - fkasumi@jfcr.or.jp; \\ Mituru Koizumi - mituru@jfcr.or.jp \\ * Corresponding author
}

Published: 17 May 2006

World Journal of Surgical Oncology 2006, 4:26 doi:10.1 186/1477-7819-4-26

This article is available from: http://www.wjso.com/content/4/I/26

(C) 2006 Mori et al; licensee BioMed Central Ltd.

This is an Open Access article distributed under the terms of the Creative Commons Attribution License (http://creativecommons.org/licenses/by/2.0), which permits unrestricted use, distribution, and reproduction in any medium, provided the original work is properly cited.
Received: 15 October 2005

Accepted: 17 May 2006

\begin{abstract}
Background: A standard intra-operative procedure for assessing sentinel lymph node metastasis in breast cancer patients has not yet been established.

Patients and methods: One hundred and thirty-eight patients with stage I breast cancer who underwent sentinel node biopsy using both imprint cytology and frozen section were analyzed.

Results: Seventeen of the 138 patients had sentinel node involvement. Results of imprint cytology included nine false negative cases (sensitivity, 47.1\%). In contrast, only two cases of false negatives were found on frozen section (sensitivity, $88.2 \%$ ). There were two false positive cases identified by imprint cytology (specificity, 98.3\%). On the other hand, frozen section had $100 \%$ specificity.
\end{abstract}

Conclusion: These findings suggest that frozen section is superior to imprint cytology for the intra-operative determination of sentinel lymph node metastasis in stage I breast cancer patients.

\section{Background}

Sentinel node biopsy has become a common surgical procedure in the treatment of breast cancer. [1] This procedure can predict the presence of nodal metastasis with high accuracy and thereby prevent morbid axillary clearance in node negative breast cancer patients. This useful technique requires the intra-operative determination of metastasis in the sentinel nodes. If sentinel node metastasis is determined intra-operatively, axillary clearance can be performed at the same time, thus obviating the need for a second operation. However, it remains unclear what should be the standard technique for intra-operative sentinel lymph node determination. Two available procedures to determine the presence of nodal metastasis are frozen section and imprint cytology. Although a few studies have shown acceptable sensitivities for frozen section applied to intra-operative diagnosis, $[2,3]$ many studies have claimed there are advantages to using imprint cytol- 
Table I: Comparison between intraoperative imprint cytology and final histological diagnosis

\begin{tabular}{|c|c|c|c|c|}
\hline & Node-positive cases at final diagnosis & \multicolumn{3}{|c|}{ Node-negative cases at final diagnosis } \\
\hline & Node-positive cases at final diagnosis & Node-negative cases a & osis & \\
\hline Positive introperative imprint cytology & 8 & 2 & & 10 \\
\hline \multirow[t]{6}{*}{ Negative introperative imprint cytology } & 9 & 119 & & 128 \\
\hline & 17 & 121 & & \\
\hline & Sensitivity & & $47.1 \%$ & \\
\hline & Specificity & & $98.3 \%$ & \\
\hline & Positive Predictive Value & & $80.0 \%$ & \\
\hline & Negative Predictive Value & & $93.0 \%$ & \\
\hline
\end{tabular}

ogy in this setting. [4-8] In this study, we compare the usefulness of imprint cytology and frozen section in the intraoperative diagnosis of sentinel node metastasis. We also review and discuss the literature regarding these techniques.

\section{Patients and methods}

From April 2000 to September 2003, we performed sentinel node mapping (SNM) in 138 breast cancer patients. Candidates for SNM had to meet pre-specified criteria, which included: a tumor size less than $2 \mathrm{~cm}$; a node-negative tumor based on palpation and ultrasonography, and no evidence of distant metastasis.

The technique of sentinel node biopsy has been described previously. $[9,10]$ Briefly, $1.5 \mathrm{mCi} / \mathrm{ml}$ of the radioactive tracer 99 mTc-rhenium sulphide or 99 mTc-phytate (Daiichi Radioisotope Laboratories, Ltd), was used. The radioactive agent was injected subdermally, close to the tumor. In all cases, a lymphoscintigraph was obtained one hour after injection. Additionally, a total of $5 \mathrm{ml}$ of vital dye (indigocallumine) was injected intradermally and into the peri-tumoral space at the operation. We had an interval of more than five minutes between the injection and the incision. Sentinel lymph nodes were identified using a hand-held Gamma probe with the assistance of stained vessels and nodes.

Sentinel nodes were evaluated by both imprint cytology and frozen section. In general, removed sentinel nodes were divided into three sections. Furthermore, we tried to section the tissue such that the sectioned plane was parallel to the plane in which the maximal section area could be obtained. When the sentinel lymph nodes were too small for trisection, they were bisected. The sectioned surface of the sentinel node was imprinted onto the surface of a slide, which was immediately immersed into a $90 \%$ alcohol solution. The slides were then stained using the Papanicolaou method. They were examined by specialized cytologists and cytopathologists. Next, the sectioned sentinel lymph nodes were sent to the laboratory, and frozen sections were made with the use of Coldtome (Sakura Fine Technical Co. Ltd., Tokyo Japan). Each frozen section was cut from one level and stained with hematoxylin and eosin (H\&E). The frozen sections were studied intra-operatively by a trained pathologist. The remaining tissue specimen from the frozen section was thawed, fixed in formalin, and embedded in paraffin. This permanent specimen, stained with $\mathrm{H} \& \mathrm{E}$, was sectioned from one level and was examined by a pathologist postoperatively. The final diagnosis of lymph node metastasis was based on histopathological evaluation. No immunohistological staining technique was used routinely.

\section{Results}

A total of 231 lymph nodes from 138 cases were evaluated by both imprint cytology and frozen section. Among these 138 patients, a total of 17 had axillary lymph node metastasis. The concordance between imprint cytology and the absolute diagnosis is shown in Table 1. According to

Table 2: Comparison between intraoperative frozen section and final histological diagnosis

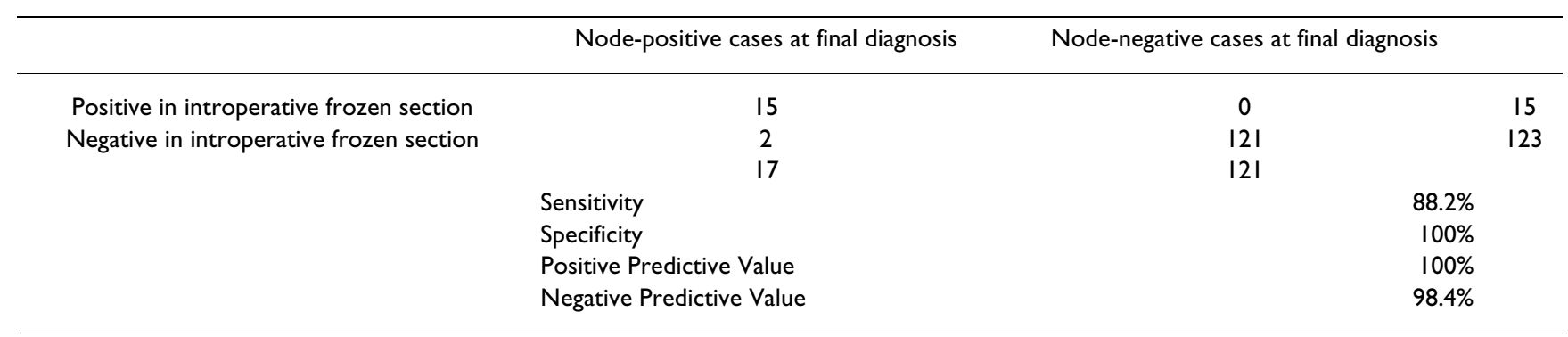


Table 3: Reported results of intraoperative imprint cytology and frozen section

\begin{tabular}{|c|c|c|c|c|c|}
\hline & \multicolumn{2}{|c|}{ imprint cytology } & \multicolumn{2}{|c|}{ frozen section } & \multirow[t]{2}{*}{ number of cases } \\
\hline & sensitivity & specificity & sensitivity & specificity & \\
\hline Present study & 47.1 & 98.3 & 88.2 & 100 & 138 \\
\hline Leidenius et al. (2003) & 68 & 99 & 83 & 99 & 375 \\
\hline Beach et al. (2003) & 69 & 100 & 54 & 100 & 32 \\
\hline Liang et al. (2003) & 62.5 & 100 & 62.5 & 100 & 20 \\
\hline Nagashima et al. (2003) & 70.3 & 99.6 & 83.8 & 100 & $\S 303$ \\
\hline Sauer et al. (2003) & 58 & 100 & 77 & 100 & $\S 429$ \\
\hline Motomura et al. (2000) & 96 & 90.8 & 52 & 100 & 101 \\
\hline van Diest et al. (1999) & 62 & 100 & 87 & 100 & 54 \\
\hline Fisher et al. (1993) & 98 & 100 & 90.2 & 100 & 50 \\
\hline
\end{tabular}

$\S$ These numbers are based on the number of examnined lymph nodes

imprint cytology there were nine false negative cases among 17 node positive patients (sensitivity, 47.1\%). On the other hand, two false positive cases were identified by imprint cytology (specificity, 98.3\%).

The agreement between frozen section and final diagnosis is shown in Table 2. Frozen section detected lymph node metastasis in 15 of 17 node positive cases (sensitivity, $88.2 \%$ ) and there were no false positive cases (specificity, $100 \%)$.

\section{Discussion}

Our study demonstrates that frozen section is superior to imprint cytology for the intra-operative diagnosis of sentinel node metastasis. Frozen section had a higher sensitivity than imprint cytology, while its high specificity was comparable to that of imprint cytology.

Several reports in the literature have compared frozen section with imprint cytology, and most of these reports have recommended imprint cytology for intra-operative sentinel lymph node determination. $[4,6,7]$ Imprint cytology is considered a rapid and convenient method with sensitivity and specificity similar to that obtained with frozen section. However, as seen in Table 3, which presents comparisons of sensitivities and specificities obtained from various reports, the sensitivity of imprint cytology is likely to be lower than that of frozen section. [6-8,11-20] Although some studies showed good sensitivities for imprint cytology, these investigators either did not investigate frozen sections $[7,12]$ or they studied more sections using imprint cytology than using frozen section. [6]

The characteristic feature of our study is that we compared imprint cytology and frozen section in an equal manner, because we studied the same sectioned surfaces using both methods. As a result, our data showed that the discrepancy in the sensitivities between these methods could be attributed mainly to sampling error associated with the imprint technique.

The evaluation of specificities in imprint cytology is also a complex issue. Generally, it is difficult to achieve a specificity of $100 \%$ using imprint cytology. There are at least two possible explanations for this difficulty. First, it is possible that benign specimens may be judged as containing malignant cells. In particular, the lobular type of breast cancer is believed to generate a false positive on imprint cytology due to the small and bland morphology of the cells. This means that some patients may undergo unnecessary axillary dissection. Unwarranted axillary clearance is clearly more problematic than a second operation for axillary dissection. The second reason is associated with micro-metastases. In cases of micro-metastases, it is possible that only imprint cytology can detect metastatic cells. However, when histopathological evaluation is required to define sentinel lymph node metastasis, the role of imprint cytology is limited.

For these reasons, we recommend frozen section rather than imprint cytology for the intra-operative diagnosis of sentinel lymph node metastasis.

We initially attempted to use imprint cytology as a modality complementary to frozen section for the intra-operative diagnosis of sentinel lymph node metastasis. Therefore, we used the Papanicolaou method for intraoperative cytological evaluation. Although this technique is time-consuming compared with other staining methods, we believe this procedure is greatly advantageous because it results in accurate evaluations.

The rate of positive sentinel node detection in patients with a sentinel node biopsy in our cohort is likely to be lower than that reported in other studies. The use of trisecting, which is nowadays inappropriate for the analysis 
of sentinel nodes, may be responsible for this result. However, we believe that ultrasonography, which was used in addition to physical examination to evaluate preoperative nodal status at our institution, contributed significantly to this consequence by allowing us to obtain more accurate nodal evaluations. The rate of positive sentinel node detection in patients who received a sentinel node biopsy should be low, because a high positive rate increases the possibility of missing axillary node metastases, both in sentinel and non-sentinel nodes. This, then, increases the likelihood of a subsequent salvage operation after swollen axillary lymph nodes become clinically apparent.

In the intra-operative diagnosis of lymph node metastasis, the management of micro-metastases is a difficult problem to solve. $[8,18]$ Several new intra-operative approaches to micrometastases have been reported. $[21,22]$ Their approaches are based on intra-operative thin sections, in which the nodes are examined thoroughly on the basis of frozen section. These time- and money-consuming methods are less likely to be introduced to routine clinical practice. The long-term prognostic impact of micro-metastasis should be established as soon as possible.

\section{Conclusion}

This study demonstrates that frozen section is superior to imprint cytology for the intra-operative diagnosis of sentinel node metastasis with respect to both sensitivity and specificity. We currently recommend using frozen section rather than imprint cytology for detecting intra-operative lymph node metastasis.

\section{Competing interests}

The author(s) declare that they have no competing interests.

\section{Authors' contributions}

KT produced the study design, conducted literature searches and drafted the manuscript. MM analyzed all data and searched the literature. MI contributed to the cytological analysis. SN, KT, MM, TI and FK contributed to surgical interventions and collected patient data. MK contributed to the analysis of nuclear medicine and collected data. All authors read and approved the final manuscript

\section{References}

I. Veronesi U, Paganelli G, Viale G, Luini A, Zurrida S, Galimberti V, Intra $M$, Veronesi $P$, Robertson $C$, Maisonneuve $P$, et al.: A randomized comparison of sentinel-node biopsy with routine axillary dissection in breast cancer. N Engl J Med 2003, 349:546-553.

2. D'Errico A, Grassigli A, Gruppioni E, Fiorentino M, Corti B, Gabusi E, Morselli-Labate AM, Grigioni WF: Thorough intraoperative analysis of breast sentinel lymph node biopsies: histologic and immunohistochemical findings. Surgery 2004, 135:248-254. discussion 255-247, 357
3. Dixon JM, Mamman U, Thomas ]: Accuracy of intraoperative frozen-section analysis of axillary nodes. Edinburgh Breast Unit team. BrJ Surg 1999, 86:392-395.

4. Lee A, Krishnamurthy S, Sahin A, Symmans WF, Hunt K, Sneige N Intraoperative touch imprint of sentinel lymph nodes in breast carcinoma patients. Cancer 2002, 96:225-231.

5. Menes TS, Tartter PI, Mizrachi H, Smith SR, Estabrook A: Touch preparation or frozen section for intraoperative detection of sentinel lymph node metastases from breast cancer. Ann Surg Oncol 2003, 10:1166-1170.

6. Motomura K, Inaji H, Komoike Y, Kasugai T, Nagumo S, Noguchi S, Koyama $\mathrm{H}$ : Intraoperative sentinel lymph node examination by imprint cytology and frozen sectioning during breast surgery. Br J Surg 2000, 87:597-60I.

7. Ratanawichitrasin A, Biscotti CV, Levy L, Crowe JP: Touch imprint cytological analysis of sentinel lymph nodes for detecting axillary metastases in patients with breast cancer. $\mathrm{Br} J$ Surg 1999, 86: I346-I348.

8. Sauer T, Engh V, Holck AM, Sorpebol G, Heim M, Furu I, Schlichting $\mathrm{E}$ : Imprint cytology of sentinel lymph nodes in breast cancer. Experience with rapid, intraoperative diagnosis and primary screening by cytotechnologists. Acta Cytol 2003, 47:768-773.

9. Koizumi M, Nomura E, Yamada Y, Takiguchi T, Tanaka K, Yoshimoto M, Makita M, Sakamoto G, Kasumi F, Ogata E: Sentinel node detection using $99 \mathrm{mTc}$-rhenium sulphide colloid in breast cancer patients: evaluation of I day and 2 day protocols, and a dosefinding study. Nucl Med Commun 2003, 24:663-670.

10. Koizumi M, Nomura E, Yamada Y, Takiguchi T, Makita M, Iwase T, Tada T, Tada K, Nishimura S, Takahashi K, et al:: Radioguided sentinel node detection in breast cancer patients: comparison of $99 \mathrm{mTc}$ phytate and $\mathbf{9 9 m T c}$ rhenium colloid efficacy. Nucl Med Commun 2004, 25: I03 I- 1037.

II. Zgajnar J, Frkovic-Grazio S, Besic N, Hocevar M, Vidergar-Kralj B, Gerljevic $A$, Pogacnik $A$ : Low sensitivity of the touch imprint cytology of the sentinel lymph node in breast cancer patients--results of a large series. J Surg Oncol 2004, 85:82-86. discussion 87

12. Ravichandran D, Kocjan G, Falzon M, Ball RY, Ralphs DN: Imprint cytology of the sentinel lymph node in the assessment of axillary node status in breast carcinoma. Eur J Surg Oncol 2004, 30:238-242.

13. Liang R, Craik J, Juhasz ES, Harman CR: Imprint cytology versus frozen section: intraoperative analysis of sentinel lymph nodes in breast cancer. ANZ J Surg 2003, 73:597-599.

14. Nagashima T, Suzuki M, Yagata H, Nikaido T, Horiuchi F, Koda K, Miyazaki M: Intraoperative cytologic diagnosis of sentinel node metastases in breast cancer. Acta Cytol 2003, 47:1028- 1032.

15. Bochner MA, Farshid G, Dodd TJ, Kollias J, Gill PG: Intraoperative imprint cytologic assessment of the sentinel node for early breast cancer. World J Surg 2003, 27:430-432.

16. Shiver SA, Creager AJ, Geisinger K, Perrier ND, Shen P, Levine EA: Intraoperative analysis of sentinel lymph nodes by imprint cytology for cancer of the breast. Am J Surg 2002, 184:424-427.

17. Van Diest PJ, Torrenga H, Borgstein PJ, Pijpers R, Bleichrodt RP, Rahusen FD, Meijer S: Reliability of intraoperative frozen section and imprint cytological investigation of sentinel lymph nodes in breast cancer. Histopathology 1999, 35: 14-18.

18. Fisher CJ, Boyle S, Burke M, Price AB: Intraoperative assessment of nodal status in the selection of patients with breast cancer for axillary clearance. $\mathrm{Br} J$ Surg 1993, 80:457-458.

19. Leidenius MH, Krogerus LA, Toivonen TS, Von Smitten KJ: The feasibility of intraoperative diagnosis of sentinel lymph node metastases in breast cancer. I Surg Oncol 2003, 84:68-73.

20. Beach RA, Lawson D, Waldrop SM, Cohen C: Rapid immunohistochemistry for cytokeratin in the intraoperative evaluation of sentinel lymph nodes for metastatic breast carcinoma. Appl Immunohistochem Mol Morphol 2003, I I:45-50.

21. Viale G, Bosari S, Mazzarol G, Galimberti V, Luini A, Veronesi P. Paganelli G, Bedoni M, Orvieto E: Intraoperative examination of axillary sentinel lymph nodes in breast carcinoma patients. Cancer 1999, 85:2433-2438.

22. Leikola JP, Toivonen TS, Krogerus LA, von Smitten KA, Leidenius MH Rapid immunohistochemistry enhances the intraoperative diagnosis of sentinel lymph node metastases in invasive lobular breast carcinoma. Cancer 2005, 104:14-19. 